\title{
Chromosome-based genomics in the cereals
}

\author{
Jaroslav Doležel ${ }^{1,2 *}$, Marie Kubaláková ${ }^{1,2}$, Etienne Paux ${ }^{3}$, Jan Bartoš ${ }^{1}$ \& Catherine Feuillet $^{3}$ \\ ${ }^{1}$ Laboratory of Molecular Cytogenetics and Cytometry, Institute of Experimental Botany, Sokolovská 6, \\ CZ-77200 Olomouc, Czech Republic; Tel: +420-585-205-852; Fax: +420-585-205-853; \\ E-mail: dolezel@ueb.cas.cz; ${ }^{2}$ Department of Cell Biology and Genetics, Palacký University, \\ Šlechtiteli 11, CZ-78371 Olomouc, Czech Republic; ${ }^{3}$ UMR ASP 1095, INRA-Université Blaise Pascal, \\ 63100 Clermont-Ferrand, France \\ * Correspondence
}

Key words: chromosome sorting, chromosome-specific BAC libraries, flow cytometry, physical mapping

\begin{abstract}
The cereals are of enormous importance to mankind. Many of the major cereal species - specifically, wheat, barley, oat, rye, and maize - have large genomes. Early cytogenetics, genome analysis and genetic mapping in the cereals benefited greatly from their large chromosomes, and the allopolyploidy of wheat and oats that has allowed for the development of many precise cytogenetic stocks. In the genomics era, however, large genomes are disadvantageous. Sequencing large and complex genomes is expensive, and the assembly of genome sequence is hampered by a significant content of repetitive DNA and, in allopolyploids, by the presence of homoeologous genomes. Dissection of the genome into its component chromosomes and chromosome arms provides an elegant solution to these problems. In this review we illustrate how this can be achieved by flow cytometric sorting. We describe the development of methods for the preparation of intact chromosome suspensions from the major cereals, and their analysis and sorting using flow cytometry. We explain how difficulties in the discrimination of specific chromosomes and their arms can be overcome by exploiting extant cytogenetic stocks of polyploid wheat and oats, in particular chromosome deletion and alien addition lines. Finally, we discuss some of the applications of flow-sorted chromosomes, and present some examples demonstrating that a chromosome-based approach is advantageous for the analysis of the complex genomes of cereals, and that it can offer significant potential for the delivery of genome sequencing and gene cloning in these crops.
\end{abstract}

\section{Introduction}

The cereals (wheat, rice and maize inter alia) are a key component of human nutrition. Their cultivation provided self-sufficiency in food and animal feed, and this was instrumental in the establishment of all the major civilizations in Central America, the Middle East, and across Asia. Cereals remain the most important of the crops, whether measured by tonnage or by market value. Bread wheat is the single largest traded crop with a global annual production exceeding 600 million tons (http://www.fao.org/), equivalent to about $100 \mathrm{~kg}$ grain per capita. To secure cereal production in the face of a growing human population, and to maintain its yield and stability across environments threatened with climate change, efficient breeding for yield, quality and resistance to biotic and abiotic stress will be paramount. Genomics has the potential to make a significant contribution to these challenges, in that it can advance our knowledge of the molecular mechanisms underlying the determination of key quantitative and qualitative agronomic traits. 
Taxonomically, all the cereals belong to the Poaceae (grass) family, distributed across four of its five main subfamilies: sorghum (Sorghum bicolor), maize (Zea mays), pearl millet (Pennisetum glaucum) and foxtail millet (Setaria italica) belong to the Panicoideae; finger millet (Eleusine indica) to the Chloridoideae; rice (Oryza sativa) to the Ehrhartoideae; and wheat (Triticum spp.), barley (Hordeum vulgare), oats (Avena sativa) and rye (Secale cereale) to the Pooideae (Table 1). The species differ markedly from one another in terms of chromosome number, ploidy level and genome size. As wheat, barley, oats and rye, and to a lesser extent maize, have very large nuclear genomes (Table 1), their chromosomes are particularly amendable to microscopic analysis and manipulation. The ability of the polyploids wheat and oats to tolerate aneuploidy has facilitated the development of a plethora of precise cytogenetic stocks, which include chromosome deletion lines, alien chromosome addition lines and substitution lines (Sears 1954, Islam et al. 1981, Joppa 1993, Jiang et al. 1994, Endo \& Gill 1996, Riera-Lizarazu et al. 1996). These features have been exploited for gene location, genetic analysis, to analyse karyotype evolution and to understand genome structure.

Individual chromosomes were first identified using their relative length, arm ratio, presence of secondary constriction(s), and behaviour during meiosis. The introduction of differential staining - particularly Giemsa C-banding - allowed unambiguous chromosome identification and the characterization of structural changes (Linde-Laursen 1988; Gill et al.
1991). Molecular cytogenetics later provided even more precise tools to identify chromosomes and chromosome segments, and to unravel chromosome organization at the molecular level (Pedersen \& Langridge 1997). Cytogenetic technologies and cytogenetic stocks continue to play an important role in the genomics era, for example by facilitating the physical mapping of molecular markers (Röder et al. 1998, Künzel et al. 2000, Kynast et al. 2004) and expressed sequences (EST) (Qi et al. 2004).

Large genomes and the polyploid state have stimulated much of recent progress in cytogenetics and genome analysis in the cereals, but in the genomics era these properties have become a liability. The sequencing of large genomes is capital-intensive, and the assembly of a genome sequence is greatly hampered, if not completely prohibited, by the presence of a significant proportion of repetitive DNA (up to $90 \%$ in the wheat genome, Paux et al. 2006). The simultaneous presence of at least two highly related genomes in the allopolyploids presents a further serious obstacle for sequence assembly. Thus many cereal genomes are not only considered intractable with respect to whole genome sequencing; the process of positional gene cloning is also made laborious and time-consuming.

In this review we survey the methods used for flow cytometry, and their application in the analysis of large and complex cereal genomes. Specifically, we describe how particular cytogenetic stocks can be exploited to dissect the genomes into their chromosome and chromosome arm components. Examples

Table 1. The major cereal crops in the Poaceae, their taxonomic classification and their genome characteristics

\begin{tabular}{|c|c|c|c|c|c|c|}
\hline Species & $\begin{array}{l}\text { Common } \\
\text { name }\end{array}$ & Subfamily & Tribe & $\begin{array}{l}\text { Chromosome } \\
\text { number }\end{array}$ & $\begin{array}{l}\text { Genome } \\
\text { size }(1 \mathrm{C})\end{array}$ & Reference \\
\hline Oryza sativa $\mathrm{L}$. & Rice & Ehrhartoideae & Oryzeae & $2 \mathrm{n}=2 \times 24$ & $490 \mathrm{Mbp}$ & Bennett \& Smith 1976 \\
\hline $\begin{array}{l}\text { Sorghum bicolour } \\
\text { (L.) Moench }\end{array}$ & Sorghum & Panicoideae & Andropogonae & $2 \mathrm{n}=2 \times=20$ & $809 \mathrm{Mbp}$ & Laurie \& Bennett 1985 \\
\hline Zea mays $\mathrm{L}$. & Maize & Panicoideae & Andropogonae & $2 \mathrm{n}=2 \times 20$ & $2793 \mathrm{Mbp}$ & Doležel \& Lucretti 1995 \\
\hline $\begin{array}{l}\text { Eleusine coracana } \\
\text { (L.) Gaertn. }\end{array}$ & Finger millet & Chloridoideae & Eragrostideae & $2 n=4 \times=40$ & $1593 \mathrm{Mbp}$ & Bennett \& Smith 1976 \\
\hline $\begin{array}{l}\text { Pennisetum glaucum } \\
\text { (L.) R.Br. }\end{array}$ & Pearl millet & Panicoideae & Paniceae & $2 n=2 \times=14$ & $2622 \mathrm{Mbp}$ & Bennett \& Smith 1976 \\
\hline Avena sativa (L.) & Oat & Poideae & Avenae & $2 \mathrm{n}=6 \times=42$ & $12961 \mathrm{Mbp}$ & Bennett \& Smith 1976 \\
\hline Hordeum vulgare (L.) & Barley & Poideae & Triticeae & $2 \mathrm{n}=2 \times=14$ & $5100 \mathrm{Mbp}$ & Doležel et al. 1998 \\
\hline Secale cereale (L.) & Rye & Poideae & Triticeae & $2 \mathrm{n}=2 \times=14$ & $7933 \mathrm{Mbp}$ & Doležel et al. 1998 \\
\hline $\begin{array}{l}\text { Triticum turgidum } \\
\text { Desf. var. durum }\end{array}$ & Durum wheat & Poideae & Triticeae & $2 n=4 \times=28$ & $12030 \mathrm{Mbp}$ & Bennett \& Smith 1976 \\
\hline Triticum aestivum (L.) & Bread wheat & Poideae & Triticeae & $2 \mathrm{n}=6 \times=42$ & $16979 \mathrm{Mbp}$ & Bennett \& Smith 1976 \\
\hline
\end{tabular}


are presented to demonstrate that a chromosomebased approach provides a powerful tool for genome analysis in the cereal crops.

\section{Flow cytometry of chromosomes}

An array of techniques has been developed over the years to allow for the microscopic analysis of higher plant chromosomes. Common to all these diverse approaches is that the chromosomes must first be immobilized on a flat surface, usually a glass slide. This requirement imposes a significant limitation on throughput and hampers the manipulation of particular chromosomes. In contrast to microscopy, flow cytometry entails holding chromosomes in a liquid suspension, which allows them to be passaged in a fastmoving narrow stream (Doležel et al. 2005a). This has the clear advantage over slide-based techniques that the analysis can be automated, and therefore progressed at a rate ranging from several hundred to well over 1000 chromosomes per second. As the chromosomes pass through an intense and focused light beam they cause the light to be scattered, and the amount of scattered light (which is readily measurable) is correlated with the size of the chromosome which caused the scattering. The effect can be magnified by staining the chromosome suspension with a DNA-binding fluorochrome. Each light pulse is converted to an electric current pulse by a photomultiplier (a photodiode in case of forward-angle light scatter) and measurements made for pulse height (the maximum value of fluorescence intensity along the chromosome), pulse area (integrated fluorescence intensity) and pulse width (related to the length of chromosome).

A series of values describing the fluorescence and light-scattering properties is generated in real time for each chromosome measured. The analysis of several thousand chromosomes generates sufficient data to characterize the karyotype. The histogram of relative chromosome fluorescence intensities is referred to as the flow karyotype. Ideally, each chromosome is represented on a flow karyotype by a single peak, but this is not the case for most plant species, since their chromosomes are generally not well enough discriminated from one another in size (Doležel et al. 2004, see also Figures 1 and 2 for examples).

Difficulties in discriminating individual chromosomes may limit the application of flow karyotyping to analyse structural chromosome changes in plants.
However, this limitation is in part compensated for by the potential of flow cytometry to physically isolate large numbers of certain specific chromosomes and/or groups of chromosomes. This sorting is effected at high speed (typically 5-30 chromosomes per second) so that purified preparations containing many copies of specific chromosomes can be generated efficiently. Flow cytometry is unique in its ability to concentrate single chromosomes in sufficient numbers, since neither microdissection nor laser capture (Potz et al. 1996, Stein et al. 1998) is able to practically generate more than a few tens of copies of any particular chromosome.

\section{The development of flow cytogenetics for cereals}

The use of flow cytometry for both chromosome analysis and sorting has been termed flow cytogenetics. Following its successful application in human and animal genetics and genomics, relevant methods have been adapted for a range of plant species (reviewed by Doležel et al. 2004). The first flow karyotyping of a cereal species was published by Wang et al. (1992), who analysed chromosomes isolated from a bread wheat cell suspension culture. Hydroxyurea (a DNA synthesis inhibitor) was used to synchronize the cell division cycle, and colchicine (a mitotic spindle poison) was applied to block the cells at mitotic metaphase.

Enzymatic hydrolysis then converted these cells to protoplasts, and mitotic chromosomes were released into solution by hypotonic lysis and mechanical shearing. The resulting chromosome suspensions were stained simultaneously with Hoechst 33258 and Chromomycin A3 fluorochromes, which bind preferentially to, respectively, AT- and GC-rich DNA regions. The resulting biparametric flow karyotypes comprised a number of peaks, but it was not clear whether these peaks represented one or more chromosomes (Wang et al. 1992), nor was the level of contamination within the peaks due to chromosome clumps and fragments determined. Nevertheless, some 100000 chromosomes were isolated from one peak and used to construct a genomic DNA library. An analysis of randomly selected clones from this library revealed an enrichment for chromosome 4A. Although only 300 recombinant clones were obtained, this first chromosome sorting in cereals demonstrated the potential of flow cytogenetics in wheat. 

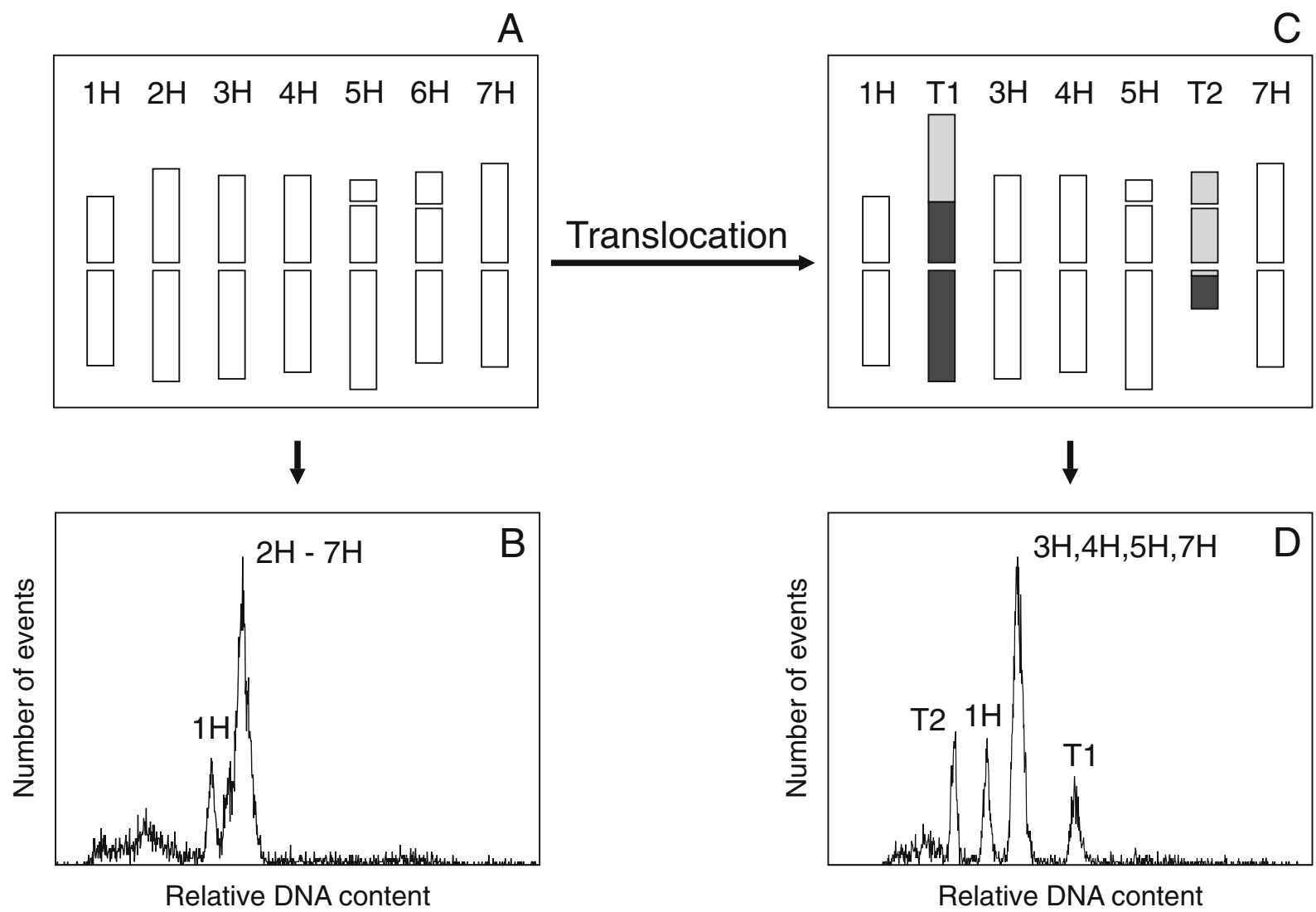

Figure 1. Alterations in the flow karyotype of barley in response to changes in chromosome size. A: In the wild-type karyotype the barley chromosomes, apart from chromosome $1 \mathrm{H}$, differ from one another only marginally in length and hence also DNA content. B: The flow karyotype of wild-type barley, in which only the chromosome $1 \mathrm{H}$ peak is well separated. The remaining six chromosomes form a composite peak and cannot be discriminated from one another. C: In barley line T2-6y, a reciprocal translocation results in a large T6HL-2HS-2HL (T1), and a small T6HS-6HL-2HS (T2) chromosome. D: The relative DNA contents of the two translocation chromosomes differ from that of other five chromosomes and both are well separated on a flow karyotype.

The outcome of the Wang et al. (1992) study highlighted some of the problems affecting similar work in other crops (reviewed by Doležel et al. 1994). Plant cells cultured in liquid suspensions are characterized by genetic instability. The cell line used by Wang et al. was later cytogenetically analysed by Schwarzacher et al. (1997), who showed it to be heterogeneous and affected by both aneuploidy and structurally aberrant chromosomes. Chromosomal heterogeneity hinders the induction of a high level of mitotic synchrony. Furthermore, the isolation of chromosomes from protoplasts requires a period during which chromosomes tend to split into sister chromatids and eventually de-condense. Although biparametric analysis using dyes differing in base-pair preference is a standard method in animal and human flow cytogenetics, in plants it does not appear to improve chromosome discrimination over what is achieved with monoparametric analysis. The most probable reason is the prevalence of dispersed repeats in plant genomes, resulting in small differences in overall AT/GC ratios among individual chromosomes (Lucretti \& Doležel 1997).

Further progress in flow cytogenetics of cereals has relied on methodological improvements achieved in other species. At first a novel protocol for chromosome isolation was introduced (Doležel et al. 1992), according to which chromosome suspensions were prepared by the mechanical homogenization of formaldehyde-fixed root tips. A mild fixation with formaldehyde prior to homogenization increased the yield of intact chromosomes. This protocol, now almost universally used in plant flow cytogenetics (Doležel et al. 2005a,b), has as its major advantages the easy handling of seedlings during the cell synchronization pro- 


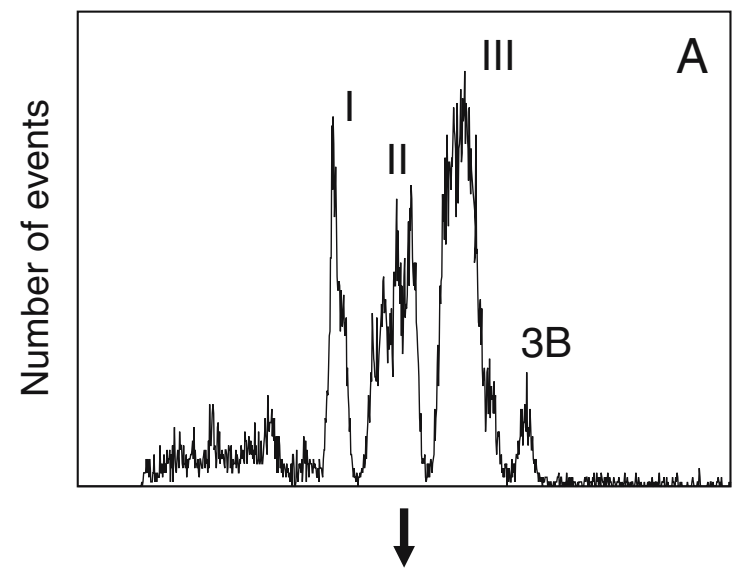

Alien chromosome addition

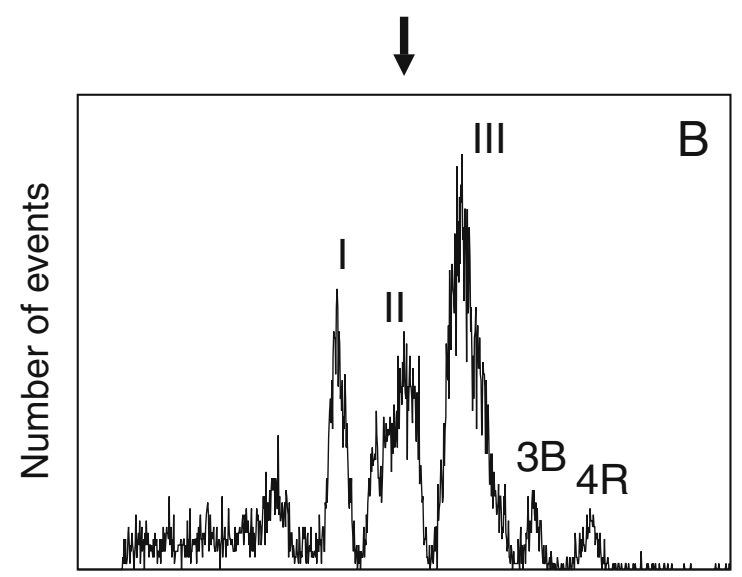

Relative DNA content

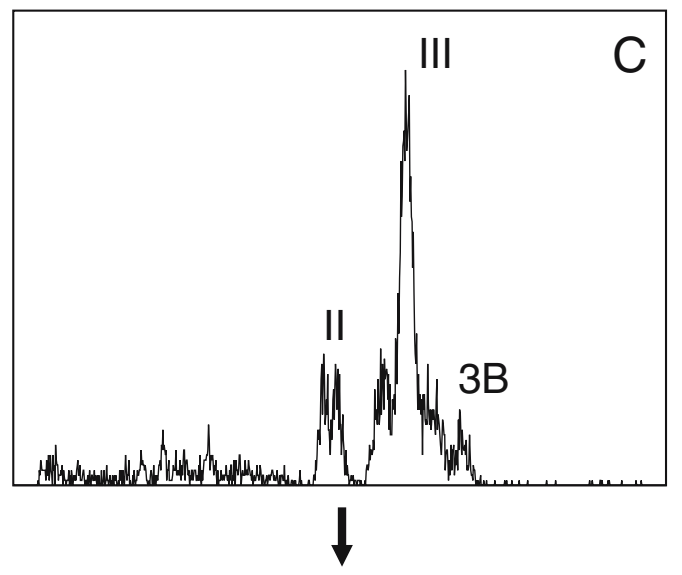

Chromosome deletion

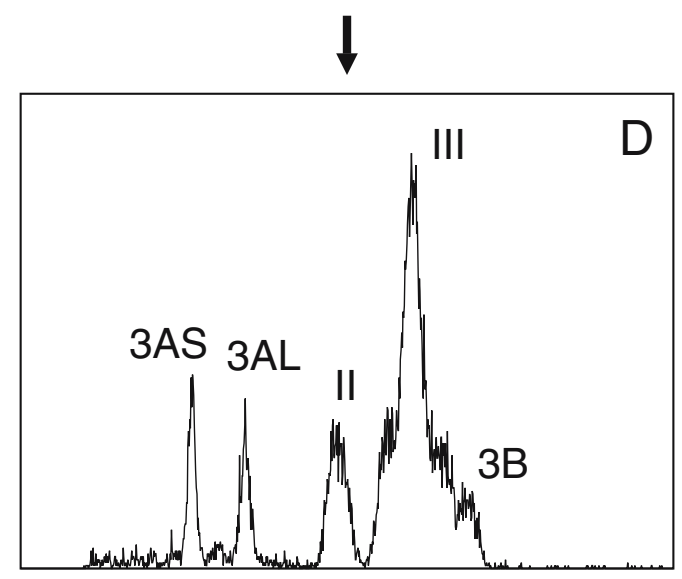

Relative DNA content

Figure 2. Changes in the flow karyotype of aneuploids, relative to that of euploid wheat. A: The flow karyotype of euploid bread wheat consists of the chromosome 3B peak, the small composite peak I containing chromosomes 1D, 4D and 6D, and the two large composite peaks II and III containing the remaining 17 chromosomes. B: The flow karyotype of a bread wheat line carrying an added pair of rye chromosome 4R. The peak representing 4R is clearly separated from the wheat chromosomes. C: The flow karyotype of euploid durum wheat consists of three peaks: chromosome 3B, a small composite peak II representing chromosomes $1 \mathrm{~A}$ and $6 \mathrm{~A}$, and a large composite peak III representing the remaining 11 chromosomes. D: The flow karyotype of double ditelosomic $3 \mathrm{~A}$ in durum wheat is characterised by additional peaks representing the short arm telosome $3 \mathrm{AS}$ and the long arm telosome $3 \mathrm{AL}$.

cedure, a high degree of synchrony, and karyological stability. The mechanical isolation eliminates the need for enzymatic hydrolysis, and the fixation both arrests the cells at the desired stage (metaphase), and makes the chromosomes less liable to mechanical shearing during the isolation, analysis and sorting processes. The second methodological improvement was pioneered by Lucretti et al. (1993) in an analysis of cytogenetic stocks of broad bean (Vicia faba L.) in which chromosome lengths had been altered by translocations. A directed choice of translocation lines allowed for individual chromosomes to be discrimi- nated by monoparametric flow karyotyping (Doležel \& Lucretti 1995). As all other attempts to improve discrimination by flow cytometry had failed, including the use of biparametric staining (Lee et al. 2000, Lucretti \& Doležel 1997, Kovářová et al. 2007) and fluorescent labelling of repetitive DNA sequences (Macas et al. 1995), the use of cytogenetic stocks, including translocations, deletions and alien additions, has emerged as the best approach to identify and sort particular chromosomes and their arms (Doležel et al. 2004). In the following, we analyse the development of flow cytogenetics in the Triticeae, and in maize and rice. 


\section{Flow cytogenetics in the Triticeae}

In a modification of the Doležel protocol for barley (Lysák et al. 1999), an average yield of $5 \times 10^{5}$ chromosomes from 50 root tips was achieved. Highresolution flow karyotypes were obtained from DAPIstained chromosome suspensions. Despite this, only chromosome $1 \mathrm{H}$ could be discriminated in the flow karyotype, with the remaining six chromosomes $(2 \mathrm{H}-7 \mathrm{H})$ forming a composite peak (Figure 1A, B). However, in some cultivars, a chromosome $2 \mathrm{H}$ peak was also resolved (Lee et al. 2000, Suchánková et al. 2006). Inspired by the work of Lucretti et al. (1993), Lysák et al. (1999) then studied a series of barley lines in which the length of some chromosomes was altered due to reciprocal translocations. At least three chromosomes could be recognized in the flow karyotypes of particular cytogenetic stocks (Figure 1C, D). The purity of the preparations of sorted chromosomes reached $97 \%$, as determined by a microscopic analysis of sorted chromosomes labelled by the PRINS (primed in-situ labelling) technique using primers recognizing GAA microsatellite motifs. The small degree of contamination was due to the rare presence of chromosome arms and chromatids of other chromosomes. Lysák et al. (1999) were also able to demonstrate the utility of sorted translocation chromosomes for the physical mapping of DNA sequences at the subchromosomal level.

A modification of Doležel's root tip method for the preparation of bread wheat chromosome suspensions was reported by Lee et al. (1997). As the formaldehyde fixation step was omitted, a rather low yield of 82000 chromosomes from 60 root tips was obtained (Gill et al. 1999). Propidium-iodide stained material produced flow karyotypes in which peaks assumed to represent chromosomes and chromatids could be resolved, but no attempt was made to identify the chromosome content of any of the individual peaks. The authors contrasted chromosomes from the wheat cultivar 'Chinese Spring' with those from a line of 'Chinese Spring' lacking chromosome arm 1DL (ditelosomic 1DS). Flow karyotypes of the latter included a peak absent in the former, assumed to be composed of the 1DS telosome, and which could be sorted with a purity exceeding $82 \%$ (Gill et al. 1999). This observation confirmed the utility of cytogenetic stocks to identify particular chromosomes. A year later, Vrána et al. (2000) reported high-resolution flow karyotyping in bread wheat, and were able to assign all 21 wheat chromosomes to four peaks of a flow karyotype, using a combination of PRINS on sorted chromosomes with primers targeted to GAA microsatellites and Afa repeats, and chromosome-specific PCR. The largest wheat chromosome (3B) formed a discrete peak and was readily sortable. The remaining 20 chromosomes clustered into the three other peaks, one of which consisted of chromosomes 1D, 4D and 6D (Figure 2A). In addition to describing the bread wheat flow karyotype, flow karyotyping was also shown to be useful for the detection of translocations between wheat chromosomes.

The flow karyotypes of a set of 58 varieties and landraces of bread wheat demonstrated the reproducibility of the technique, and were used to detect structural chromosome changes and chromosome polymorphisms (Kubaláková et al. 2002). The wheat/ rye translocation $1 \mathrm{BL} \cdot 1 \mathrm{RS}$ is present in many modern wheat cultivars and its presence generates a diagnostic change in flow karyotype. However, the discriminating peak was not sufficiently resolved to allow effective sorting of the translocated chromosome. Other translocation chromosomes, in particular $5 \mathrm{BL} \cdot 7 \mathrm{BL}$ and 4AL-4AS-5BL, produced flow karyotype peaks which were discrete enough to permit sorting. However, the most important breakthrough was that almost all the wheat telosomes (with the exception of 3BL and 5BL), which are maintained in stable cytogenetic stocks covering most of the 42 chromosome arms (Sears 1954), could be recognized against the background of the rest of the genome, and sorted. The 3BL and $5 \mathrm{BL}$ arms were isolated from stocks carrying them as isochromosomes.

The flow karyotype of tetraploid (durum, or macaroni) wheat was characterized by Kubaláková et al. (2005). Due to the absence of D-genome chromosomes, only three peaks were generated, one containing chromosome $3 \mathrm{~B}$, a small peak (1A and 6A), and a large peak containing the remaining 11 chromosomes (Figure 2C). Assignment of chromosomes to flow karyotype peaks was achieved by applying FISH (fluorescence in-situ hybridization) to sorted chromosomes using probes targeting the GAA microsatellite motif, $p S c 119.2$ and $A f a$ repeats. Although durum wheat has a smaller number of chromosomes than bread wheat (14 against 21 ), only one chromosome (3B) could be unequivocally sorted. Nonetheless, by exploiting the double ditelosomic lines of durum wheat, in which each chromosome in turn is represented by a pair of telosomes (Joppa 1993), it was possible to isolate each 
A- and B-genome chromosome arm (Kubaláková et al. 2005, and Figure 2D). As the flow karyotype of durum wheat was less complex than that of bread wheat, the telosome peaks were better defined, which in turn resulted in the recovery of a $90-98 \%$ purity level in sorted preparations.

Rye has the largest nuclear genome (1C $7.9 \mathrm{Gbp})$ among the diploid Triticeae species. Methods for the preparation of chromosome suspensions and flow karyotyping were developed by Kubaláková et al. (2003). The karyotypes consisted of a composite peak representing chromosomes 2R-7R. Chromosome $1 \mathrm{R}$ formed a shoulder on the left side of the composite peak in most cultivars. However, in some cultivars (e.g. 'Imperial'), the $1 \mathrm{R}$ peak was fully resolved and the chromosome could be sorted. Interestingly, the flow karyotype of rye was similar to that of barley, whose nuclear genome is somewhat smaller (1C $~ 5.1 \mathrm{Gbp}$ ). This implies that, during the speciation of these crop species, each chromosome expanded in a proportional fashion. Flow karyotyping was also able to detect both inter-chromosome translocations and the presence of supernumerary B chromosomes.

Although direct flow sorting could only successfully isolate a single rye chromosome, recourse to wheat-rye chromosome addition lines overcame this limitation. In these lines each pair of rye chromosomes in turn is present in a bread wheat background, and the added rye chromosomes $2 \mathrm{R}-7 \mathrm{R}$ are sufficiently different in size to any of the wheat chromosomes to allow for their straightforward identification and sorting (Kubaláková et al. 2003, and Figure 2B).
The sensitivity of the profiles was such that the dosage of the added rye chromosome in a population could be deduced from its flow karyotype based on relative height of the rye peak. More recently, Šafár et al. (2006) exploited a wheat-rye ditelosome addition line to purify chromosome arm 1RS. The same approach could be used to sort independently those chromosome arms of rye, which are shorter than chromosome $1 \mathrm{D}$, the shortest in the bread wheat complement.

Of the intact barley chromosomes, to date only chromosome $1 \mathrm{H}$, and in certain genotypes chromosome $2 \mathrm{H}$, could be flow-sorted (Figure 1). The success of the alien addition line approach for rye chromosomes stimulated Suchánková et al. (2006) to explore a similar strategy in barley. Each of the chromosome arms of barley present in the wheat-barley ditelosomic addition lines was sortable, although no attempt was made to sort the arms of $1 \mathrm{H}$, as this chromosome can be isolated directly from the barley flow karyotype. In any case, given the sterility of the telosomic $1 \mathrm{HL}$ addition (Islam \& Shepherd 1990, 2000), only the short arm of $1 \mathrm{H}$ would have been accessible via this route.

\section{A flow cytogenetic toolbox for the Triticeae}

Strategies have now been developed to dissect the genomes of the major Triticeae crops into their individual chromosomes and chromosome arms (Doležel et al. 2005b), relying on the availability of appropriate wheat cytogenetic stocks and alien chromosomal structural variants. As shown in Table 2,

Table 2. The possibilities for dissecting the nuclear genomes of the Triticeae crops by flow sorting

\begin{tabular}{|c|c|c|c|c|}
\hline \multirow{3}{*}{ Karyotype } & \multicolumn{4}{|c|}{ Chromosomes and chromosome arms that can be isolated by flow sorting* } \\
\hline & Barley & Rye & Durum wheat & Bread wheat \\
\hline & $1 \mathrm{C} \sim 5.1 \mathrm{Gbp}$ & $1 \mathrm{C} \sim 7.9 \mathrm{Gbp}$ & $1 \mathrm{C} \sim 12 \mathrm{Gbp}$ & $1 \mathrm{C} \sim 17 \mathrm{Gbp}$ \\
\hline Wild-type & $1 \mathrm{H}(12.2 \%)$ & $1 \mathrm{R}(12.7 \%)$ & 3B $(8.6 \%)$ & $3 \mathrm{~B}(5.9 \%)$ \\
\hline Telosomic lines & - & - & All arms $(2.1-5.2 \%)$ & $\begin{array}{l}\text { All arms, } \\
\quad \text { except 3BL, 5BL }(1.3-3.2 \%) \text {; }\end{array}$ \\
\hline Isochromosome lines & - & - & - & 3BL, 5BL $(3.3 \%, 3.4 \%)$ \\
\hline $\begin{array}{l}\text { Wheat chromosome } \\
\text { addition lines }\end{array}$ & - & 2R-7R (13.2-16.6\%) & - & - \\
\hline $\begin{array}{l}\text { Wheat telosome } \\
\text { addition lines }\end{array}$ & Arms of $2 \mathrm{H}-7 \mathrm{H}(5.9-9.0 \%)$ & $1 \mathrm{RS}(5.6 \%)$ & - & - \\
\hline
\end{tabular}

* In some genotypes additional chromosomes can be isolated. The values in parentheses show the relative portions of the nuclear genome represented by the smallest and the largest chromosomes and/or chromosome arms that can be sorted. Relative chromosome (arm) lengths were taken from Marthe \& Künzel (1994) for barley, Schlegel et al. (1987) for rye, Venora et al. (2002) for durum wheat and Gill et al. (1991) for bread wheat. 
the Triticeae genomes can be dissected in this way into segments as small as $1.3 \%$ of the total genome, translating to about $220 \mathrm{Mbp}$ in bread wheat or about half of the size of the fully sequenced rice genome (IRGSP 2005; Goff et al. 2002; Yu et al. 2002). Should segments smaller than chromosome arms be needed, it is possible to generate these via the activity of gametocidal chromosomes (Shi \& Endo 1997, Yoshino et al. 1998). Thus, it may be possible in the future to isolate, via flow sorting, portions of the Triticeae genomes smaller than a chromosome arm.

\section{Flow cytogenetics of maize}

Lee et al. (1996) were the first to report a flow cytometric analysis of the chromosomes of maize. Chromosome suspensions were prepared from root meristems using a method modified from that of Doležel et al. (1992). Flow karyotypes of propidiumiodide stained samples matched the profile predicted from relative chromosome sizes, and suggested the possibility of discriminating and sorting two (chromosomes 1 and 10) of the 10 maize chromosomes. However, no attempt was made to assign chromosomes to flow karyotype peaks. Later, flow karyotypes of a range of maize inbred and hybrid lines were produced (Lee et al. 2002), revealing variation in the number and position of chromosome peaks, which presumably reflects different genome sizes and the presence of heterochromatic chromosomal knobs. At least five different chromosome types could be discriminated and sorted from five different maize lines, although confirmation of the purity of these preparations has not yet been forthcoming. The difficulties experienced in sorting the maize chromosomes prompted the exploration of an alternative strategy, particularly focusing on the oat-maize chromosome addition lines developed by Riera-Lizarazu et al. (1996). In these lines a single maize chromosome or chromosome pair is present in a hexaploid oat background. The oat-maize chromosome-9 addition line produced a flow peak corresponding to maize chromosome 9 and the chromosome was sorted at a level of purity exceeding 90\% (Li \& Arumuganathan 2001).

\section{Flow cytogenetics of oats}

In order to use an oat-maize addition line, $\mathrm{Li} \&$ Arumuganathan (2001) had first to optimize a pro- tocol for the preparation of chromosome suspensions from oat root tips. Although a flow karyotype of hexaploid oats cv. 'Starter-1' has been presented, it has not been clarified whether this allows for the sorting of any individual oat chromosomes. Thus, at present the potential of flow cytogenetics for dissecting the genome of oats remains to be assessed.

\section{Flow cytogenetics of rice}

Rice has a small genome, which has proved to be amenable to sequencing and gene cloning. Recently, the genomes of the two rice subspecies indica and japonica have been separately sequenced (Goff et al. 2002, Yu et al. 2002, IRGSP 2005), and rice is universally considered to be the appropriate model for cereal genomics (Xu et al. 2005). Lee \& Arumuganathan (1999) optimized a protocol for isolating chromosomes from synchronized rice root tips. Although peaks thought to represent individual chromosomes were observed, the low resolution achieved prevented an estimation of how many, if any, chromosomes could be sorted. However, even a predicted flow karyotype showed that only one chromosome could be discriminated (Lee \& Arumuganathan 1999). The rapid progress made in rice genomics makes flow cytogenetics unlikely to play an important role in rice compared to that in the large genome cereals.

\section{The integration of flow cytogenetics and genomics}

The sequencing of higher plant genomes is advancing rapidly and it is expected that 12 plant genomes will have been sequenced by 2006 (Paterson 2006, see also http://www.jgi.doe.gov). Candidates for sequencing are chosen largely on the basis of (small) genome size in order to avoid technical problems associated with the sequencing of complex genomes. For the latter, a strategy of sample sequencing has been proposed (Paterson 2006). Although this represents a large cost saving over whole genome shotgun sequencing, it suffers from the drawback that short DNA sequences cannot be readily assembled to reconstruct the full genome. Assembly becomes possible only when the sample sequences can be anchored to an extant physical genome map (Meyers et al. 2004).

Several methods have been applied to create physical maps, in which the genome is described by 
an assembly of adjacent or overlapping segments. Two in particular of these are worth mentioning in the context of flow cytometry, although neither has been applied on any large scale to date. HAPPY mapping (Thangavelu et al. 2003) relies on the co-segregation of markers on randomly sheared genomic DNA fragments, while optical mapping (Aston et al. 1999) arrays digested DNA molecules and seeks to derive ordered restriction maps from images of the digested molecules. High molecular weight DNA is required for both these methods, and this can be prepared from flow-sorted cereal chromosomes. Thus sorted chromosomes could represent the basis of physical maps related a priori to a specific chromosome or chromosome arm.

Almost all genome-wide physical maps have been generated by the ordering of BAC (bacterial artificial chromosome) clones, via DNA fingerprinting (Luo et al. 2003). These maps serve to anchor short sequences to a genome scaffold, enable clone-by-clone sequencing and positional gene cloning. The assembly of physical maps in the large genome cereals is hampered by the large number of clones required to achieve full genome coverage, by the ubiquity of dispersed repeats, and by polyploidy. Thus the $9 \times$ coverage BAC library of bread wheat consists of $1.2 \times 10^{6}$ clones (Allouis et al. 2003) and although it is technically possible to fingerprint such a large numbers of clones, the assembly of reliable contigs and the construction of a physical map represents an enormous challenge.

\section{Chromosome-specific BAC libraries}

An elegant solution to the size problem is to create BAC libraries specific for small and defined genome segments, and this represents an area in which chromosome sorting can play a crucial role. Until 2004 it was unclear whether large-insert DNA libraries could be constructed from flow-sorted chromosomes, and three critical developments were seen as necessary to achieve this goal. These were: (1) the ability to sort individual chromosomes/chromosome arms in sufficiently large quantities, as discussed above; (2) the preparation of unsheared DNA from sorted chromosome preparations in a form suitable for BAC cloning (Šimková et al. 2003); and (3) the development of an efficient protocol for BAC cloning (Chalhoub et al. 2004). Thus, a subgenomic BAC library specific for bread wheat chromosomes 1D, 4D and 6D was generated by Janda et al. (2004), and one for bread wheat chromosome 3B by Šafár et al. (2004) (Table 3). The 3B library was constructed from a sample of $1.8 \times 10^{6}$ flow-sorted chromosomes and represents the first BAC library to be produced from a specific eukaryotic chromosome.

Most of the wheat and barley chromosomes can only be sorted as chromosome arms, rather than as whole chromosomes. As the arms represent smaller parts of the genomes than chromosomes, they must be sorted in higher numbers to provide enough DNA for BAC library construction. A 1BS BAC library has been generated recently from $6 \times 10^{6}$ purified chromosome arms, sorted from ditelosomic 1BS, a line lacking chromosome arm 1BL (Janda et al. 2006). Representing just $1.9 \%$ of the wheat genome (Table 3), the library achieves a $14.5 \times$ coverage of $1 B S$. An even deeper $(17 \times)$ coverage was generated in a 1RS BAC library (Šafár et al. 2006). This chromosome arm is present in many modern wheat cultivars in the form of a 1BL·1RS chromosome, as its presence is associated with improved agronomic performance. Notably, good coverage is achieved in these chromosome (arm) BAC libraries from a relatively modest number of clones (typically $<10^{5}$ ), making the libraries practical to maintain and screen.

Table 3. BAC libraries already constructed from flow-sorted chromosomes of Triticeae species

\begin{tabular}{|c|c|c|c|c|c|c|c|}
\hline \multirow[b]{2}{*}{ Species } & \multicolumn{3}{|l|}{ Chromosome } & \multicolumn{4}{|l|}{ BAC library } \\
\hline & $\begin{array}{l}\text { Chromosome } \\
\text { number }\end{array}$ & $\begin{array}{l}\text { Genome } \\
\text { fraction }\end{array}$ & $\begin{array}{l}\text { Molecular } \\
\text { size }(\mathrm{Mb})\end{array}$ & $\begin{array}{l}\text { Number of } \\
\text { clones }\end{array}$ & $\begin{array}{l}\text { Mean insert } \\
\text { size }(\mathrm{kb})\end{array}$ & Coverage & Reference \\
\hline T. aestivum & $1 \mathrm{D}, 4 \mathrm{D}, 6 \mathrm{D}$ & $11.6 \%$ & 1969 & 87168 & 85 & $3.4 \times$ & Janda et al. (2004) \\
\hline T. aestivum & $3 \mathrm{~B}$ & $5.9 \%$ & 995 & 67986 & 103 & $6.2 \times$ & Šafáŕ et al. (2004) \\
\hline T. aestivum & 1BS & $1.9 \%$ & 315 & 65280 & 82 & $14.5 \times$ & Janda et al. (2006) \\
\hline S. cereale & $1 \mathrm{RS}$ & $5.6 \%$ & 442 & 103680 & 83 & $17.0 \times$ & Šafáŕ et al. (2006) \\
\hline T. aestivum & $3 \mathrm{AS}$ & $2.1 \%$ & 361 & 55296 & 80 & $12 \times$ & Šimková et al. (in preparation) \\
\hline
\end{tabular}




\section{The utility of subgenomic BAC libraries}

About $10 \mathrm{Mb}$ of wheat A-, B- and D-genome BAC sequence have been sequenced and analysed to date (Paux et al. 2006). However, most of this sequence relates to short segments (mean of about $200 \mathrm{~kb}$ of contiguous sequence) and originates mostly from gene-rich and telomeric regions of the genome. Thus it provides a rather limited and unrepresentative picture of the overall genome. Wheat's large genome size and the presence of three homoeologous genomes have hindered large-scale and representative analyses to date. A suggested means to generate sufficient random sequence to allow an unbiased assessment of genome sequence content and organization is target $\mathrm{BAC}$ end sequences (BES). Thus in maize, Messing et al. (2004) produced $475000 \mathrm{BES}$, equivalent to $307 \mathrm{Mb}$ or $12 \%$ of the total genome. To achieve a similar representation in bread wheat, 3.3 million BES would be needed, and it would also be necessary to determine the chromosomal origin of each sequence in order to identify features specific to each of the constituent genomes. Alternatively, on the assumption that the genomes remained unchanged during the process of polyploidization, the three donor species could be analysed separately. However, as dramatic rearrangements are thought to have occurred following polyploidization (Feldman \& Levy 2005), the ancestral genomes probably represent at best only an approximate model of the hexaploid genome, not mentioning difficulties in identifying the B-genome donor. Therefore, the most efficient means to expose the composition and evolution of the homoeologous genomes will be to analyse the individual chromosomes of bread wheat, using BAC libraries from sorted chromosomes.

The power of such a chromosome-based approach is well illustrated by current investigations of wheat chromosome 3B. The $67968 \mathrm{BAC}$ clones of the 3B BAC library have now been fingerprinted and assembled to construct a physical map of the chromosome (unpublished data). In the first phase of this assembly, Paux et al. (2006) generated 3306 contigs, which were used to select clones for BAC end sequencing and the characterization of chromosome structure. In all, 10752 clones were sequenced, generating about $11 \mathrm{Mb}$ of sequence evenly distributed along the chromosome. This sequence comprised $86 \%$ repetitive elements, $1.2 \%$ coding sequence (corresponding to 6000 genes) and $13 \%$ unknown sequence. A number of novel repetitive sequences was also uncovered. By means of a comparison with $2.9 \mathrm{Mb}$ of random sequence from Aegilops tauschii, the D-genome donor, it appears that the large size of the $\mathrm{B}$ genome chromosomes is due to their higher content of repetitive elements. The transposable element (TE) families most likely responsible for the evolutionary differential expansion of the wheat genomes were also identified (Paux et al. 2006). Clearly, the availability of a purified preparation of a single chromosome was critical to reveal these features of the bread wheat B genome, results hard to achieve by performing an analysis at the whole genome level.

\section{Perspectives in genome sequencing and gene cloning using subgenomic $\mathrm{BAC}$ resources}

The establishment of a physical map of the bread wheat genome represents the first step towards the goal of sequencing the genome. In addition, a physical map of each of the 21 wheat chromosomes will serve to accelerate efforts to isolate, using a map-based approach, the hundreds of genes and QTL that have been genetically mapped to date. One of the most timeconsuming steps in such cloning projects remains the establishment of a physical contig spanning the target. Chromosome-specific BAC libraries should allow a rapid drafting of the relevant physical map with contigs ranging from $200 \mathrm{~kb}$ to $>1 \mathrm{Mb}$. Once the target region has been identified on a high-density genetic map it is a straightforward procedure to then identify the candidate BAC contig(s), establish contigs spanning the gene of interest, and sequence the region to identify candidate genes. Thus the International Wheat Genome Sequencing Consortium (IWGSC, www.wheatgenome. org) plans now to construct chromosome arm-specific BAC libraries from cv. 'Chinese Spring' for which a genomic BAC library is also available (Allouis et al. 2003). If a particular allele of interest is not present in 'Chinese Spring', a pooled BAC library can be rapidly produced (Isidore et al. 2005) from a genotype carrying the trait of interest and screened with probes extracted from the 'Chinese Spring' physical map.

\section{The targeted isolation of molecular markers}

In addition to the construction of physical maps, other genomics applications of flow-sorted chromosomes deserve attention. Fine-scale genetic mapping requires the saturation of the genetic maps in the 
region of the target, and thus the development of many localized molecular markers. Such markers can be retrieved from chromosome-specific DNA libraries constructed from just hundreds or thousands of chromosomes, which are then amplified either by DOP-PCR (Telenius et al. 1992) or by whole genome amplification with DNA polymerase phi29 (Dean et al. 2001). This strategy has been explored in both non-cereal crops (Arumuganathan et al. 1994, Požárková et al. 2002, Román et al. 2004) and rye (R. Kofler, personal communication). The major advantage of a chromosome-based approach is that the chromosomal specificity of each candidate marker can be verified, prior to genetic mapping, by a simple PCR from a template of flow-sorted chromosomes (Požárková et al. 2002). A further option for the targeted isolation of markers from chromosome-derived DNA is offered by DArT technology (Wenzl et al. 2004), based on microarray hybridization and designed to detect and type DNA variation at several hundred genomic loci in parallel. Although to date DArT has only been applied on genomic DNA, there is no reason to doubt that it would function equally well on a target of chromosomal DNA.

Effective markers can also be developed from BES. Paux et al. (2006) combined this idea with a chromosome-based approach in order to saturate the genetic map of wheat chromosome $3 \mathrm{~B}$, specifically targeting ISBP (insertion site-based polymorphisms) markers. Junctions between TE and non-TE sequences, which represent the site of insertion of a TE, were identified from 20000 BES produced from the chromosome 3B BAC library. From 2000 of these junctions, which have proven to be highly polymorphic, 58 primer pairs were designed to assess the potential of ISBP for mapping in wheat. About $67 \%$ of these were assignable to bins defined by the standard wheat deletion line set, while the remainder were informative in various segregating populations. By extrapolation therefore, as many as 1300 BES-derived markers could be added to the chromosome 3B map. A similar approach is being used to saturate the genetic map of rye chromosome $1 \mathrm{R}$, focusing on microsatellite sequence markers (Bartoš et al., in preparation).

\section{The molecular organization of cereal chromosomes}

Localization of DNA sequences on chromosomes is commonly performed by FISH or PRINS to mitotic metaphase spreads from root tip meristems. However, practical considerations limit the number of analysable metaphase plates and so restrict the analytical throughput achievable. Furthermore, the sensitivity and specificity of FISH and PRINS are often compromised by interference from cell wall and cytoplasmic debris. The application of FISH and PRINS to sorted chromosomes (Lucretti et al. 1993, Kubaláková et al. 2001, Li \& Arumuganathan 2001) provides the opportunity to screen hundreds of chromosomes per slide, with better resolution, sensitivity and throughput. In an analysis of flow-sorted rye B chromosomes, Kubaláková et al. (2003) were able to demonstrate rare translocations between $\mathrm{A}$ and $\mathrm{B}$ chromosomes. As these occurred at a frequency of just $0.5 \%$, their discovery by conventional screening of metaphase squashes would have required the processing of hundreds of slides. FISH-based analyses of sorted wheat chromosomes have allowed for both the uncovering of polymorphism in GAA banding patterns (Kubaláková et al. 2002), and the construction of a molecular karyotype of durum wheat (Kubaláková et al. 2005).

The ability of FISH to resolve two adjacent DNA sequences depends on a combination of genome size and the degree of chromosome condensation. The minimum range of resolution is from $5-10 \mathrm{Mb}$ for mitotic metaphase chromosomes, $0.2-1 \mathrm{Mb}$ for meiotic pachytene chromosomes and 1-5 kb for stretched DNA fibres (de Jong et al. 1999). For some applications the resolution of the latter is higher than necessary but the resolution of pachytene FISH is too low, especially in large genome cereals, and particularly in polyploid species. Thus there is a need for a method which can provide an intermediate level of resolution. This was achieved by Valárik et al. (2004), who were able to extend, by up to $100 \times$, the length of flow-sorted barley, rye and wheat chromosomes. The application of FISH to these stretched chromosomes gave a spatial resolution of up to $70 \mathrm{~kb}$.

Chromosome painting is a technique central to human cytogenetics. Its principle is to label each chromosome in a specific manner, using FISH with a hybridization cocktail of many probes. A critical factor for the success of this technique is probe specificity. Despite many attempts to develop painting in large genome plants, it has been difficult to prevent crosshybridization of probes, even though where they have been extracted from microdissected chromosome arms (e.g. wheat chromosome 5BL) or sub-arm segments (e.g. the distal quarter of the long arm of barley trans- 


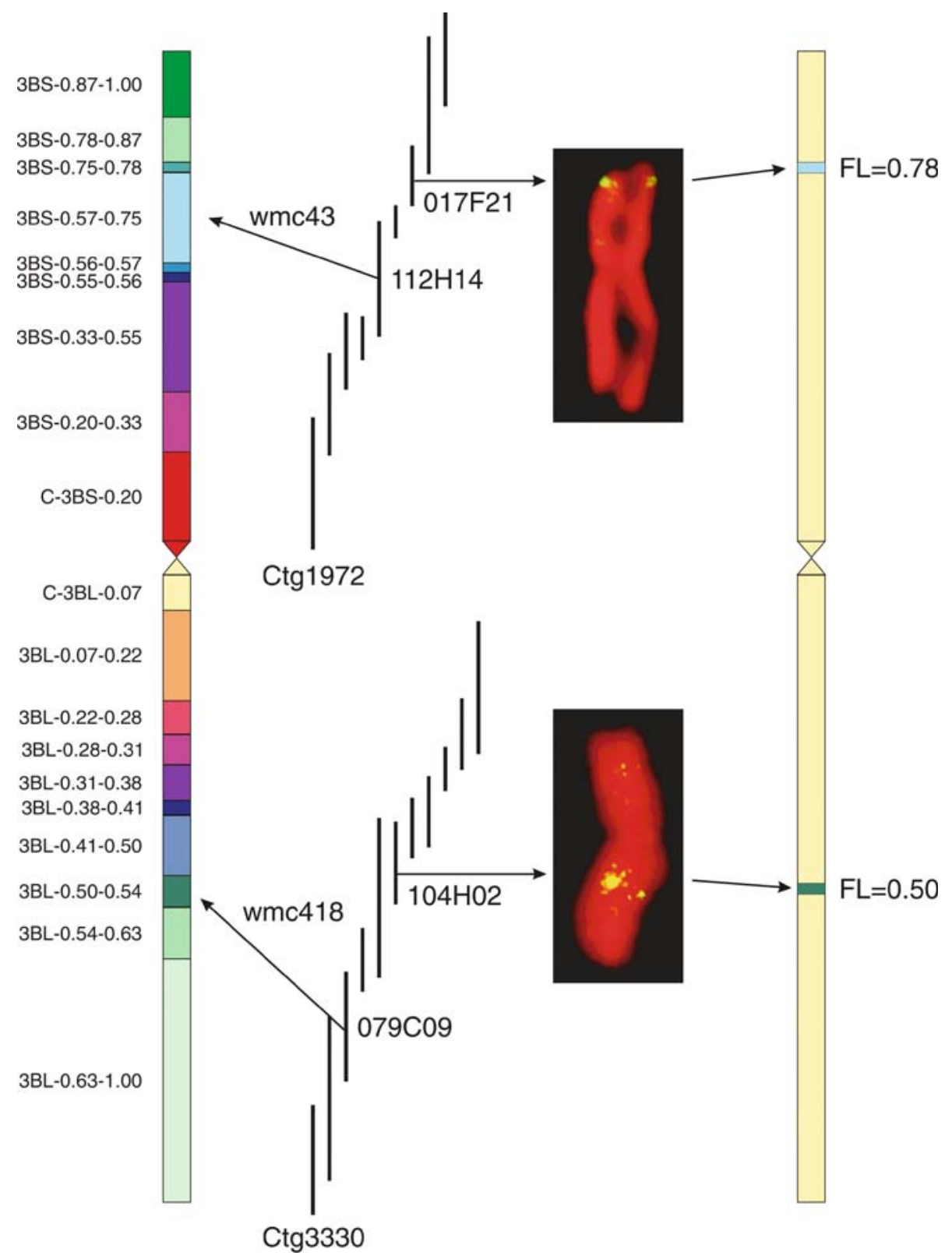

Figure 3. The integration of physical contigs and cytogenetic maps for wheat chromosome 3B. In the bin map of chromosome 3B (left), the bins are labelled by the fraction lengths (FL) of their borders (FL represents the percent of the chromosome arm length). Two BAC contigs (Ctg1972 and Ctg3330) were anchored to specific bins by means of the genetically mapped microsatellite markers wmc43 and wmc418. From each of the contigs a BAC clone containing low amounts of repetitive DNA was selected and localized by FISH to flow-sorted chromosome 3B. The cytogenetic map on the right shows the position of two BAC clones on chromosome 3B expressed as mean FL (average of 10 analyses). The analysis confirmed the position of the contigs on the chromosome and improved the precision of their location. 
location T7HS·7HL-5HL, Fuchs et al. 1996), or from flow-sorted barley chromosome $1 \mathrm{H}$ (Cremer et al., unpublished observation). This failure has been explained as being the result of a combination of the production of a weak signal from low-copy sequences and the dominance of dispersed repeats across the whole genome (Fuchs et al. 1996). Interestingly, similar arguments were used to explain the failure of biparameric flow karyotyping (Lucretti \& Doležel 1997, Kovárová et al. 2007).

Isolated broad bean chromosomes have been shown to be suitable for the analysis of chromosome structure using scanning electron microscopy and immunostaining of chromosomal antigens (Schubert et al. 1993). This approach has been little explored in the cereals. However, a set of kinetochore proteins was successfully localized using immunofluorescent staining of isolated chromosomes of barley (ten Hoopen et al. 2000) in a study which also demonstrated the widespread conservation of SKP1 and CBF5 protein domains in plants. Immunostaining of sorted barley telochromosome variants of $7 \mathrm{HS}$ revealed that centromere-specific proteins and phosphorylated histone H3 were localized in the centromeric region even though the barley-specific satellite sequence was absent (Nasuda et al. 2005). This surprising result led to the conclusion that, for the assembly of the kinetochore, centromeric repeats are neither sufficient nor necessary.

\section{Closing the circle ...}

The parallel development of flow-sorting and BAC technology offers an exciting opportunity to develop specific probes for FISH and PRINS, and to apply these to sorted chromosomes. The high throughput and sensitivity of FISH on sorted chromosomes are ideal for its application as a cytogenetic mapping tool. The construction of physical maps is aided by prior localization of a BAC contig to a chromosomal segment, by orienting contigs with respect to the centromere and telomere, and by enabling the estimation of the size of gaps between adjacent contigs. This approach has been used to confirm the position of BAC contigs in wheat chromosome 3B (Bartoš et al., unpublished observations, and Figure 3), while FISH has been applied to flow-sorted wheat and rye chromosomes to localize clones selected from chromosomespecific BAC libraries (Šafář et al. 2004, Janda et al.
2004, 2006). The initial success rate for the mapping of clones to a specific locus was low, due to interference from dispersed repeats, but this difficulty was largely overcome by the development of low-copy BAC subclones as FISH probes (Janda et al. 2006). The success of this approach relied on an ability to localize DNA sequences of about $2 \mathrm{~kb}$ to sorted chromosomes.

\section{Future prospects}

Practical methods for chromosome analysis and sorting to dissect the genomes of important cereals with complex genomes such as wheat, barley, rye and maize are now available. The possibility of constructing chromosome- and chromosome armspecific BAC libraries holds out a promising prospect for the further development of genomics in these species, as they not only simplify the process of physical map construction and positional cloning, but also provide a viable strategy to sequence chromosomes one at a time. Moreover, the division of these large genomes into manageable portions helps to structure an international collaboration aiming to tackle large and polyploid genomes such as that of wheat. Thus, together with other applications of sorted chromosomes, flow cytogenetics now offers a versatile toolbox for the analysis of chromosome organization and function of large genome species. Along with the technical advantages of flow cytogenetics, it is important to keep in mind that the equipment cost and the operator technical skill level required are both high. For this reason it would be sensible to support the establishment of a small number of specialized laboratories capable of producing a volume of flow-sorted chromosomes and chromosome-specific genome resources sufficient to meet the requirements of downstream user laboratories. Once again therefore, flow cytogenetics may act as a stimulant for international collaboration.

\section{Acknowledgements}

We are grateful to our colleagues, Pavlína Kovářová, MSc, Dr Jan Šafár, Dr Hana Šimková, and Pavla Suchánková, MSc, for sharing unpublished data. This work was supported by the Czech Science Foundation (grant awards 521/06/P412, 521/05/0257 and 521/06/1723), the Ministry of Education, Youth and Sports of the Czech Republic (grant award ME884 
and LC06004), INTAS (grant award 03-51-5908) and INRA grants. We thank http://www.smartenglish.co. uk for linguistic advice in the preparation of this manuscript.

\section{References}

Allouis S, Moore G, Bellec A et al. (2003) Construction and characterisation of a hexaploid wheat (Triticum aestivum L.) BAC library from the reference germplasm 'Chinese Spring'. Cereal Res Commun 31: 331-338.

Arumuganathan K, Martin GB, Telenius H, Tanksley SD, Earle ED (1994) Chromosome 2-specific DNA clones from flowsorted chromosomes of tomato. Mol Gen Genet 242: 551-558.

Aston C, Mishra B, Schwartz DC (1999) Optical mapping and its potential for large-scale sequencing projects. Trends Biotech $\mathbf{1 7}$ : 297-302.

Bennett MD, Smith J (1976) Nuclear DNA amounts in angiosperms. Phil Trans $R$ Soc B 274: 227-274.

Chalhoub B, Belcram H, Caboche M (2004) Efficient cloning of plant genomes into bacterial artificial chromosome (BAC) libraries with larger and more uniform insert size. Plant Biotechnol J 2: 181-188.

Dean FB, Nelson JR, Giesler TL, Lasken RS (2001) Rapid amplification of plasmid and phage DNA using phi29 DNA polymerase and multiply-primed rolling circle amplification. Genome Res 11: 1095-1099.

de Jong JH, Fransz P, Zabel P (1999) High resolution FISH in plants - techniques and applications. Trends Plant Sci 4: 258-263.

Doležel J, Lucretti S (1995) High-resolution flow karyotyping and chromosome sorting in Vicia faba lines with standard and reconstructed karyotypes. Theor Appl Genet 90: 797-802.

Doležel J, Č́halíková J, Lucretti S (1992) A high-yield procedure for isolation of metaphase chromosomes from root tips of Vicia faba L. Planta 188: 93-98.

Doležel J, Greilhuber J, Lucretti S, et al. (1998) Plant genome size estimation by flow cytometry: Inter-laboratory comparison. Ann Bot 82 (Suppl. A): 17-26.

Doležel J, Lucretti S, Schubert I (1994) Plant chromosome analysis and sorting by flow cytometry. Crit Rev Plant Sci 13: 275-309.

Doležel J, Kubaláková M, Bartoš J, Macas J (2004) Flow cytogenetics and plant genome mapping. Chromosome Res 12: 77-91.

Doležel J, Kubaláková M, Bartoš J, Macas J (2005a) Chromosome flow sorting and physical mapping. In Meksem K, Kahl G, eds., The Handbook of Plant Genome Mapping. Genetic and Physical Mapping. Weinheim: Wiley-VCH Verlag GmbH \& Co. KGaA, pp. 151-171.

Doležel J, Kubaláková M, Suchánková P et al. (2005b) Flow cytogenetic analysis of the wheat genome. In Tsunewaki K, ed., Frontiers of Wheat Bioscience. Hundredth Memorial Issue of Wheat Information Service. Yokohama: Kihara Memorial Yokohama Foundation for the Advancement of Life Sciences, pp. 3-15.

Endo TR, Gill BS (1996) The deletion stocks of common wheat. $J$ Hered 87: 295-307.
Feldman M, Levy AA (2005) Allopolyploidy - a shaping force in the evolution of wheat genomes. Cytogenet Genome Res 109: 250-258.

Fuchs J, Houben A, Brandes A, Schubert I (1996) Chromosome 'painting' in plants - A feasible technique? Chromosoma 104: 315-320.

Gill BS, Friebe B, Endo TR (1991) Standard karyotype and nomenclature system for description of chromosome bands and structural aberrations in wheat (Triticum aestivum L.). Genome 34: $830-839$.

Gill KS, Arumuganathan K, Le JH (1999) Isolating individual wheat (Triticum aestivum) chromosome arm by flow cytometric analysis of ditelosomic lines. Theor Appl Genet 98: 1248-1252.

Goff SA, Ricke D, Lan TH et al. (2002) A draft sequence of the rice genome (Oryza sativa L. ssp japonica). Science 296: 92-100.

International Rice Genome Sequencing Project (2005) The mapbased sequence of the rice genome. Nature 436: 793-800.

Isidore E, Scherrer B, Bellec A et al. (2005) Direct targeting and rapid isolation of BAC clones spanning a defined chromosome region. Func Integr Genomics 5: 97-103.

Islam AKMR, Shepherd KW, Sparrow DHB (1981) Isolation and characterization of euplasmic wheat-barley chromosome addition lines. Heredity 46: 161-174.

Islam AKMR, Shepherd KW (1990) Incorporation of barley chromosomes into wheat. In Bajaj YPS, ed., Biotechnology in Agriculture and Forestry 13: Wheat. Berlin: Springer-Verlag, pp. 128-151.

Islam AKMR, Shepherd KW (2000) Isolation of a fertile wheatbarley addition line carrying the entire barley chromosome $1 \mathrm{H}$. Euphytica 111: 145-149.

Janda J, Bartoš J, Šafár J et al. (2004) Construction of a subgenomic BAC library specific for chromosomes 1D, 4D and 6D of hexaploid wheat. Theor Appl Genet 109: 1337-1345.

Janda J, Šafáŕ J, Kubaláková M et al. (2006) Novel resources for wheat genomics: BAC library specific for the short arm of chromosome 1B. Plant J 47: 977-986.

Jiang J, Friebe B, Gill BS (1994) Recent advances in alien gene transfer in wheat. Euphytica 73: 199-212.

Joppa LR (1993) Chromosome engineering in tetraploid wheat. Crop Sci 33: 908-913.

Kováŕová P, Navrátilová A, Macas J, Doležel J (2007) Chromosome analysis and sorting in common vetch (Vicia sativa L.) using flow cytometry. Biol Plant 51: 43-48.

Kubaláková M, Vrána J, Č́íhalíková J, Lysák MA, Doležel J (2001) Localisation of DNA sequences on plant chromosomes using PRINS and C-PRINS. Methods Cell Sci 23: 71-82.

Kubaláková M, Vrána J, Číhalíková J, Šimková H, Doležel J (2002) Flow karyotyping and chromosome sorting in bread wheat (Triticum aestivum L.). Theor Appl Genet 104: 1362-1372.

Kubaláková M, Valárik M, Bartoš J (2003) Analysis and sorting of rye (Secale cereale L.) chromosomes using flow cytometry. Genome 46: 893-905.

Kubaláková M, Kovárová P, Suchánková P (2005) Chromosome sorting in tetraploid wheat and its potential for genome analysis. Genetics 170: 823-829.

Künzel G, Korzun L, Meister A (2000) Cytologically integrated physical restriction fragment length polymorphism maps for the 
barley genome based on translocation breakpoints. Genetics 154: $397-412$.

Kynast RG, Okagaki RJ, Galatowitsch MW (2004) Dissecting the maize genome by using chromosome addition and radiation hybrid lines. Proc Natl Acad Sci USA 101: 9921-9926.

Laurie DA, Bennett MD (1985). Nuclear DNA content in the genera Zea and Sorghum Intergeneric, interspecific and intraspecific variation. Heredity 55: 307.

Lee JH, Arumuganathan K (1999) Metaphase chromosome accumulation and flow karyotypes in rice (Oryza sativa L.) root tip meristem cells. Mol Cells 9: 436-439.

Lee JH, Arumuganathan K, Kaeppler SM, Kaeppler HF, Papa CM (1996) Cell synchronization and isolation of metaphase chromosomes from maize (Zea mays L) root tips for flow cytometric analysis and sorting. Genome 39: 697-703.

Lee JH, Arumuganathan K, Yen Y, Kaeppler S, Kaeppler H, Baenziger PS (1997) Root tip cell cycle synchronization and metaphase-chromosome isolation suitable for flow sorting in common wheat (Triticum aestivum L.). Genome 40: 633-638.

Lee JH, Arumuganathan K, Chung YS et al. (2000) Flow cytometric analysis and chromosome sorting of barley (Hordeum vulgare L.). Mol Cells 10: 619-625.

Lee JH, Arumuganathan K, Kaeppler SM et al. (2002) Variability of chromosomal DNA contents in maize (Zea mays L.) inbred and hybrid lines. Planta 215: 666-671.

Li L, Arumuganathan K (2001) Physical mapping of 45S and 5S rDNA on maize metaphase and sorted chromosomes by FISH. Hereditas 134: 141-145.

Linde-Laursen I (1988) Giemsa C-banding of barley chromosomes. VI. Localization of breakpoints in 70 reciprocal translocations. Hereditas 108: 65-76.

Lucretti S, Doležel J (1997) Bivariate flow karyotyping in broad bean (Vicia faba). Cytometry 28: 236-242.

Lucretti S, Doležel J, Schubert I, Fuchs J (1993) Flow karyotyping and sorting of Vicia faba chromosomes. Theor Appl Genet 85: 665-672.

Luo MC, Thomas C, You FM et al. (2003) High-throughput fingerprinting of bacterial artificial chromosomes using the SNaPshot labeling kit and sizing of restriction fragments by capillary electrophoresis. Genomics 82: 378-389.

Lysák MA, Č́íhalíková J, Kubaláková M, Šimková H, Künzel G, Doležel J (1999) Flow karyotyping and sorting of mitotic chromosomes of barley (Hordeum vulgare L.). Chrom Res 7: 431-444.

Macas J, Doležel J, Gualberti G, Pich U, Schubert I, Lucretti S (1995) Primer-induced labeling of pea and field bean chromosomes in situ and in suspension. BioTechniques 19: 402-408.

Marthe F, Künzel G (1994) Localization of translocation breakpoints in somatic metaphase chromosomes of barley. Theor Appl Genet 89: 240-248.

Messing J, Bharti AK, Karlowski WM et al. (2004) Sequence composition and genome organization of maize. Proc Natl Acad Sci USA 101: 14349-14354.

Meyers BC, Scalabrin S, Morgante M (2004) Mapping and sequencing complex genomes: let's get physical! Nature Rev Genet 5: 578-581.

Nasuda S, Hudakova S, Schubert I, Houben A, Endo TR (2005) Stable barley chromosomes without centromeric repeats. Proc Natl Acad Sci USA 102: 9842-9847.
Paterson AH (2006) Leafing through the genomes of our major crop plants: strategies for capturing unique information. Nat Rev Genet 7: 174-184.

Paux E, Roger D, Badaeva E et al. (2006) Characterizing the composition and evolution of homoeologous genomes in hexaploid wheat through BAC-end sequencing on chromosome 3B. Plant $J$ 48: 463-474.

Pedersen C, Langridge P (1997) Identification of the entire chromosome complement of bread wheat by two-colour FISH. Genome 40: 589-593.

Potz H, Schubert V, Houben A, Schubert I, Weber WE (1996) Aneuploids as a key for new molecular cloning strategies: development of DNA markers by microdissection using Triticum aestivum-Aegilops markgrafii chromosome addition line B. Euphytica 89: 41-47.

Požárková D, Koblížková A, Román B (2002) Development and characterization of microsatellite markers from chromosome 1specific DNA libraries of Vicia faba. Biol Plant 45: 337-345.

Qi LL, Echalier B, Chao SA et al. (2004) Chromosome bin map of 16,000 expressed sequence tag loci and distribution of genes among the three genomes of polyploid wheat. Genetics 168 : 701-712.

Riera-Lizarazu O, Rines HW, Phillips RL (1996) Cytological and molecular characterization of oat $\times$ maize partial hybrids. Theor Appl Genet 93: 123-135.

Román B, Šatovič Z, Požárková D et al. (2004) Development of a composite map in Vicia faba, breeding applications and future prospects. Theor Appl Genet 108: 1079-1088.

Röder MS, Korzun V, Gill BS, Ganal MW (1998) The physical mapping of microsatellite markers in wheat. Genome 41 : 278-283.

Šafár J, Bartoš J, Janda J et al. (2004) Dissecting large and complex genomes: flow sorting and BAC cloning of individual chromosomes from bread wheat. Plant J 39: 960-968.

Šafár J, Šimková H, Suchánková P et al. (2006) A novel resource for genomics of rye and wheat: BAC library specific for the short arm of chromosome 1R (1RS). In: Abstracts of the International Conference 'Plant and Animal Genome XIV'. San Diego: Sherago International, p. 115.

Schlegel R, Melz G, Nestrowicz R (1987) A universal reference karyotype in rye, Secale cereale L. Theor Appl Genet 74: $820-826$.

Schubert I, Doležel J, Houben A, Scherthan H, Wanner G (1993) Refined examination of plant metaphase chromosome structure at different levels made feasible by new isolation methods. Chromosoma 102: 96-101.

Schwarzacher T, Wang ML, Leitch AR, Miller N, Moore G, Heslop-Harrison JS (1997) Flow cytometric analysis of the chromosomes and stability of a wheat cell-culture line. Theor Appl Genet 94: 91-97.

Sears ER (1954) The aneuploids of common wheat. Missouri Agric Exp Stn Res Bull 572: 1-58.

Shi F, Endo TR (1997) Production of wheat-barley disomic addition lines possessing an Aegilops cylindrica gametocidal chromosome. Genes Genet Syst 72: 243-248.

Šimková H, Č́íhalíková J, Vrána J, Lysák MA, Doležel J (2003) Preparation of high molecular weight DNA from plant nuclei and chromosomes isolated from root tips. Biol Plantarum 46: 369-373. 
Stein N, Ponelies N, Musket T, McMullen M, Weber G (1998) Chromosome micro-dissection and region-specific libraries from pachytene chromosomes of maize (Zea mays L.). Plant $J$ 13: 281-289.

Suchánková $\mathrm{P}$, Kubaláková $\mathrm{M}$, Kovárová $\mathrm{P}$ et al. (2006) Dissection of the nuclear genome of barley by chromosome flow sorting. Theor Appl Genet 113: 651-659.

Telenius H, Carter NP, Bebb CE, Nordenskjöld M, Ponder BAJ, Tunnacliffe A (1992) Degenerate oligonucleotide-primed PCR: general amplification of target DNA by a single degenerate primer. Genomics 13: 718-725.

ten Hoopen R, Manteuffel R, Doležel J, Malysheva L, Schubert I (2000) Evolutionary conservation of kinetochore protein sequences in plants. Chromosoma 109: 482-489.

Thangavelu M, James AB, Bankier A, Bryan GJ, Dear PH, Waugh R (2003) HAPPY mapping in a plant genome: reconstruction and analysis of a high-resolution physical map of a $1.9 \mathrm{Mbp}$ region of Arabidopsis thaliana chromosome 4. Plant Biotech J 1: 23-31.

Valárik M, Bartoš J, Kovářová P, Kubaláková M, de Jong H, Doležel J (2004) High-resolution FISH on super-stretched flowsorted plant chromosomes. Plant J 37: 940-950.
Venora GS, Blangiforti MR, Castiglione D et al. (2002) Chromatin organisation and computer aided karyotyping of Triticum durum Desf. cv. Timilia. Caryologia 55: 91-98.

Vrána J, Kubaláková M, Šimková H, Č́íhalîková J, Lysák MA, Doležel J (2000) Flow-sorting of mitotic chromosomes in common wheat (Triticum aestivum L.). Genetics 156: 2033-2041.

Wang ML, Leitch AR, Schwarzacher T, Heslop-Harrison JS, Moore G (1992) Construction of a chromosome-enriched HpaII library from flow-sorted wheat chromosomes. Nucleic Acids Res 20: $1897-1901$.

Wenzl P, Carling J, Kudrna D et al. (2004) Diversity arrays technology (DArT) for whole-genome profiling of barely. Proc Natl Acad Sci USA 101: 9915-9920.

$\mathrm{Xu}$ YB, McCouch SR, Zhang QF (2005) How can we use genomics to improve cereals with rice as a reference genome? Plant Mol Biol 59(1): 7-26.

Yoshino M, Nasuda S, Endo TR (1998) Detection of terminal deletions in barley chromosomes by the PCR-based method. Genes Genet Syst 73: 163-168.

Yu J, Hu SN, Wang J et al. (2002) A draft sequence of the rice genome (Oryza sativa L. ssp indica). Science 296: 79-92. 